\title{
Behcet's disease
}

\author{
Authors: Jagdish R Nair ${ }^{\mathrm{A}}$ and Robert ] Moots ${ }^{\mathrm{B}}$
}

Behçet's disease (BD) is a chronic relapsing and remitting vasculitis of unknown aetiology. It has the capacity to affect almost all organ systems because of its potential to involve both arteries and veins of all sizes, resulting in significant organ-threatening morbidity and mortality. Traditionally known as the 'silk road' disease, it has a worldwide occurrence. The aetiopathological mechanisms of disease development in BD remain poorly understood, but genome wide studies show human leukocyte antigen and non-human leukocyte antigen associations. Environmental influences and genetic factors may have a role in the aetiopathogenetic mechanisms that lead to development of the disease, indicating the autoimmune and auto-inflammatory nature of BD. The evidence base for treatment is limited but new knowledge is emerging and current treatment options range from symptomatic treatment, through to non-biological and biological immunosuppressive drugs, to cover the spectrum of clinical manifestations.

\section{Introduction}

Behçet's disease (BD) is a rare but severely debilitating vasculitis, which typically manifests as mucocutaneous disease with orogenital ulcers and skin lesions; ${ }^{1}$ however, involvement of the musculoskeletal system, eye, nervous system, gastrointestinal tract, vascular beds, urogenital tract and cardio-pulmonary system can lead to significant morbidity and mortality.

There is often a long delay between the onset of symptoms and diagnosis of BD because of the variable, and sometimes intermittent, symptoms, the need for exclusion of mimics, the lack of a specific blood test and marker for the disease and, sadly, a general lack of awareness of this condition.

\section{Epidemiology}

BD occurs worldwide but clusters are found mainly along the 'silk road' with highest prevalence in Turkey (approximately 80-370 cases per 100,000), ${ }^{2}$ Japan and Iran, and lower

Authors: A consultant rheumatologist, National Behcet's Syndrome Centre of Excellence, Aintree University Hospital, Liverpool, UK; B professor of rheumatology, University of Liverpool and director, National Behcet's Syndrome Centre of Excellence, Aintree University Hospital, Liverpool, UK prevalence in North American and northern European populations. In the UK, there is an estimated 0.64 cases per $100,000 .{ }^{3}$ While the commonest age group affected is those aged $20-40$ years, BD is also seen in children and older patients. Incidence is higher in males in the high prevalence areas of Turkey and Middle East, but sex distribution is variable in other counties. The disease is usually severe in young adult men.

$\mathrm{BD}$ is mainly sporadic, but clustering can occur in families. The aetiology remains unknown, but a combination of genetic and environmental factors may play a role. The HLA-B51 genetic marker is found in around $60 \%$ of BD patients. ${ }^{4}$ Genome-wide association studies (GWAS) have identified HLA-B51 and HLA ERAP1 (endoplasmic reticulum aminopeptidases) as $\mathrm{BD}$ susceptibility genes. ${ }^{5-7}$

\section{Key points}

Behçet's disease is a chronic inflammatory disease of unknown aetiology that can affect all body organ systems because of inflammatory effects on arteries and veins of all sizes

Diseases that cause orogenital ulcerations have to be excluded by investigations before confirming BD; sexual infections and inflammatory bowel diseases are the main mimics

Treatment is tailored to individual patient clinical manifestations and involves the use of various symptomatic measures and disease modifying non-biological and biological therapies

The use of anticoagulation in thrombosis is controversial and immunosuppressive therapy is recommended for management of venous and arterial thrombosis

Eye disease is common and needs careful assessment and treatment because of the high risk of visual loss

Multidisciplinary pharmacological and non-pharmacological management is vital

KEYWORDS: Behcet's disease, biological drugs, diseasemodifying drugs, immunosuppression, vasculitis 


\begin{tabular}{ll}
$\begin{array}{l}\text { Table 1. The International Study Group criteria } \\
(1990)^{10}\end{array}$ & $\begin{array}{l}\text { Features } \\
\text { Criteria }\end{array}$ \\
$\begin{array}{ll}\text { Recurrent oral ulcerations } & \begin{array}{l}\text { Minor aphthous, major aphthous } \\
\text { or herpetiform ulceration observed } \\
\text { by physician or patient, which } \\
\text { recurred at least three times in } \\
\text { one 12-month period }\end{array} \\
\text { Plus any two of the following: } & \\
\text { Recurrent genital ulceration } & \begin{array}{l}\text { Aphthous ulceration or scarring } \\
\text { observed by physician or patient }\end{array} \\
\text { Eye lesion } & \begin{array}{l}\text { Anterior uveitis, posterior uveitis } \\
\text { or cells in vitreous on slit lamp } \\
\text { examination or retinal vasculitis } \\
\text { observed by ophthalmologist } \\
\text { Erythema nodosum observed } \\
\text { by physician or patient, }\end{array} \\
\text { pseudofolliculitis or papulopustular } \\
\text { lesions, or acneiform nodules } \\
\text { observed by physician } \\
\text { Read by a physician at 24-48 hours. }\end{array}$ \\
\hline
\end{tabular}

In a susceptible individual, as yet unknown environmental factors, which may include microbial exposure and cellular and humoral immunity, play a pathogenic role. Proinflammatory cytokine cascade, inflammatory responses, relapsing and remitting course and treatment responses to immunosuppressive medications in $\mathrm{BD}$ indicate the disease has an autoinflammatory-autoimmune nature.

\section{Classification criteria}

The International Criteria for Behçet's disease (ICBD), ${ }^{8}$ International Team for the Revision of the International Criteria for Behcet's Disease (ITR-ICBD, 2013) ${ }^{9}$ and the International Study Group criteria (Table 1$)^{10}$ are the classification criteria currently in use. The International Study Group criteria ${ }^{10}$ are less sensitive but more specific than the ICBD criteria (Table 2) ${ }^{8}$ and ITR-ICBD criteria. ${ }^{9}$ A diagnosis of $\mathrm{BD}$ is made based on the clinical picture - defined by characteristic features after exclusion of relevant differential diagnoses by appropriate assessments and investigations.

Table 2. The International Criteria for Behçet's disease $(2006)^{8}$

\begin{tabular}{ll} 
Symptom & Score $^{*}$ \\
Genital aphthosis & Two points \\
Ocular lesions & Two points \\
Oral aphthosis & One point \\
Skin lesions & One point \\
Vascular lesions & One point \\
Pathergy & One point \\
\hline *3 or more points satisfy criteria for Behçet's disease &
\end{tabular}

*3 or more points satisfy criteria for Behçet's disease

\section{Clinical manifestations}

BD can affect potentially all organ systems because of its propensity to affect all arteries and veins.

Oral and genital ulcers are the hallmarks of the disease, seen in up to $97 \%$ and $60-90 \%$ of patients, respectively. The oral ulcers in $\mathrm{BD}$ are similar to the common benign recurrent aphthous stomatitis, can vary in size from minor (less than $10 \mathrm{~mm}$ in size) to major (greater than $10 \mathrm{~mm}$ ) to herpetiform ulcers (pin head to 1-3 mm), and may scar. Importantly, these ulcers are painful and may affect eating and swallowing.

Genital ulcers can affect any part of genitourinary tract and can scar. They are common in the scrotum in male patients and the vulva in female patients, and can take weeks to heal. Although BD does not affect fertility directly, painful recurrence of genital ulcerations in either males or females may limit the capacity for sexual intercourse.

It is important to exclude other causes of oral ulcers, such as infection, cyclical neutropenia, medication, inflammatory bowel disease (IBD), vitamin B12 deficiencies, rheumatic diseases (eg systemic lupus erythematosus), autoinflammatory diseases, PFAPA (periodic fever, aphthosis, pharyngitis and adenitis), Sweet's syndrome and mimics, like bullous dermatosis. It is also important to exclude other possible causes of genital ulcerations: sexual infections, non-sexually acquired genital ulcerations (which may be reactive to infections), IBD, drug reactions, trauma and neoplasms. Complex aphthosis is a condition of oral and genital ulcers in the absence of other features of BD. Oral and genital ulcers also occur in MAGIC (mouth and genital ulcers with inflamed cartilage) syndrome, cyclical neutropenia and as adverse drug effects.

Cutaneous disease manifests with papulonodular lesions, erythema nodosum-like lesions, acneiform rashes, pseudofolliculitis, pyoderma gangenosum and occasionally erythema multiforme-like rashes. Pathergy, an eruption of a papulopustular lesion at the site of needle injury within 24-48 hours, is not exclusive to BD - it also occurs in Sweet's syndrome and pyoderma gangernosum. Cutaneous lesions may be commonly associated with arthritis in BD.

Gastrointestinal BD can feature ulceration throughout the tract, but mainly in the mouth, oesophagus, ileo-caecal and ascending colon, and may be indistinguishable from IBD. Diarrhoea, bleeding, abdominal pain and fever are common presenting features and, occasionally, complications like fistula and bowel perforation occur. Orofacial granulomatosis, intestinal biopsy showing granulomata and paper cut genital ulcers are suggestive of IBD. Endoscopic and radiological investigations will be required to map the disease.

Neuro-Behçet's disease (NBD) is a serious complication, which affects up to $10 \%$ of patients and may cause parenchymal and non-parenchymal disease. ${ }^{11}$ Parenchymal disease, which affects the cerebrum, brainstem, cerebellum and spinal cord, can result in intra-axial cranial neuropathy, ophthalmopresis, pyramidal disease, focal and multifocal cerebral lesions, and various brainstem syndromes depending on the extent of neuraxial involvement. Non-parenchymal disease includes cerebral venous sinus thrombosis, intracranial hypertension and meningitis, which may be recurrent. However, the commonest neurological symptom is migraine, which is similar to non-BD migraine. Radiological investigations, including magnetic resonance imaging (MRI), computerised tomography (CT), magnetic 
resonance angiography (MRA), computerised tomographic venography (CTV), magnetic resonance venography (MRV), cerebrospinal fluid studies and electroencephalogram, might be required in appropriate cases. Magnetic resonance diffusion imaging with magnetic resonance spectroscopy may have a role in imaging in NBD but requires large studies to validate its use.

Vascular BD is unique in affecting both arterial and venous vascular system of all sizes and is a major cause of morbidity and mortality. The arterial disease is less prevalent than venous disease. Arterial lesions resulting from inflammation cause aneurysm, ulcerations, thrombosis and stenosis, whereas, the venous disease mainly manifests as venous thrombosis and thrombophlebitis. The thrombus is usually tightly adherent to the vessel wall and may not embolise. Appropriate imaging of the vascular system with Doppler ultrasound studies, CT, CTV, MRI, MRA and MRV will be necessary. CT angiography gives good anatomical details but has limited use in early large vessel disease and MRA provides details of structural changes but correlation with clinical disease activity is not always good. MRA may be useful for following up the structural changes in blood vessels, such as an increase or decrease in size of aneurysm after treatment, and may be preferred to computerised tomography angiography because of the lack of radiation risks. ${ }^{18} \mathrm{~F}$-fludeoxyglucose positron emission tomography with CT or magnetic resonance localisation may be useful in assessment of clinical disease activity as in other large vessel vasculitis but may be modified by immunosuppressive treatment.

Arthralgia is a common symptom in BD. Oligoarthritis, monoarthritis and polyarthritis can be indistinguishable from other inflammatory arthritis, like psoriatic or rheumatoid arthritis, but is usually non-erosive and non-deforming. Enthesial inflammation can sometimes be a predominant feature. Fibromyalgia is a common cause of pain in BD.

Eye disease in $\mathrm{BD}$ is a major cause for concern as, if inadequately treated, it represents a major cause of visual loss - particularly in young males. There is a spectrum of ocular manifestations (up to $70 \%$ of patients with $\mathrm{BD}$ are affected): anterior uveitis, pars planitis, posterior uveitis, retinal vasculitis, scleritis, episcleritis, thrombosis of retinal artery and vein and optic neuritis. BD may cause ocular pain, eye redness, visual disturbances, photophobia and an estimated visual loss of approximately $10-20 \%$ at 5 years. ${ }^{12}$ Visual charting (visual fields and LogMAR visual acuity scores), ophthalmological examination, optical coherence tomography and, occasionally, visual evoked potentials are essential for both diagnosing disease and monitoring response to therapy.

Pregnancy outcome is generally favourable with flares noted only in one in six pregnancies. There are some reports of miscarriage, ${ }^{13}$ preterm labour and neonatal deaths but there is no evidence that this is different to a healthy population ${ }^{14}$ or that BD affects fertility. A 2015 study showed that BD did not negatively influence feto-maternal outcome. ${ }^{15}$

Hugh-Stovin syndrome, ${ }^{16}$ symptoms for which can include fever, chest pain, deep vein thrombosis, thrombophlebitis and pulmonary aneurysms, may present like BD and it is as yet not known whether this syndrome correctly lies within the spectrum of BD.

Fatigue is a severe accompaniment of $\mathrm{BD}$, similar to other inflammatory diseases. Unfortunately, fatigue does not always respond to successful treatment of the disease.

Uncommon manifestations of BD are listed in Box 1.

\section{Box 1. Uncommon manifestations in Behçet's}

\section{disease}

Cardiac - coronary vasculitis, pericarditis, myocarditis, endocarditis, coronary aneurysms, valvular disease and arrhythmias

Pulmonary - pulmonary opacities, haemorrhage, vasculitis, pleural effusions, pulmonary aneurysms, pulmonary hypertension and fibrosing mediastinitis

Renal - mild renal impairment, glomerulonephritis, interstitial nephritis, renal artery aneurysm and amyloidosis

Other associations - MAGIC (mouth and genital ulcers with inflamed cartilage) represents features of relapsing chondritis and mucosal ulcers

Sweet's syndrome - fever, nodular rashes, and mouth and genital ulcers

\section{Approach to the diagnosis}

A careful history and examination of all relevant systems is required, with differential diagnoses excluded by appropriate investigations (Box 2). Particularly, other systemic rheumatic diseases (eg systemic lupus erythematosus), IBD, coeliac disease, drug exposure (methotrexate, nicorandil etc), sexually transmitted diseases (herpes virus, HIV, gonorrhoea, chlamydia etc) and non-sexually acquired infection-related ulcers, cyclical neutropenia, vitamin deficiencies (B12) and Sweet's syndrome may present with manifestations that mimic BD. The confirmation of diagnosis is based on appropriate clinical symptoms, signs and exclusion of differential diagnoses. A multidisciplinary specialist assessment is mandatory to reach a diagnosis.

\section{Management}

Because of the complex nature of the disease and multisystem involvement, a holistic approach to management is required with involvement of relevant specialists. A combined pharmacological and non-pharmacological approach to management is essential, as for any chronic disease. With the absence of large randomised controlled clinical trials to evaluate the effects of various interventions in $\mathrm{BD}$, treatment is driven by recommendations of expert bodies like EULAR, with consensus statements. ${ }^{17}$

The basic tenet of treatment is to use the most effective and least toxic treatment. Various disease-modifying drugs used in $\mathrm{BD}$ are listed in Table 3 and the newer drugs, which need more evidence, are listed in Box 3. ${ }^{18-22}$ Mucosal ulcer treatment should reduce pain, duration of ulceration and ensure healing - plus, in the long term, suppress recurrence. Topical treatment provides mucosal barriers to promote healing. Oral hygiene and avoidance of sodium lauryl sulphate-containing toothpaste, an irritant, is important. Topical corticosteroids in various formulations are used. Betamethasone sodium phosphate $500 \mu \mathrm{g}$ tablets or prednisolone $5 \mathrm{mg}$ dissolved in 10-20 mL saline and held in the mouth for 3 minutes as a mouthwash, hydrocortisone oromucosal tablets applied close to the ulcer, and beclometasone dipropionate inhaler 50-100 $\mu$ g sprayed twice daily on the oral mucosa are effective topical treatment of oral ulcers. The antibiotics tetracycline $(250 \mathrm{mg}$ in $10 \mathrm{~mL}$ of water) or doxycycline 


\section{Box 2. Investigations for Behçet's disease}

\author{
Routine \\ > Full blood count, renal, liver, bone profile \\ $>$ Inflammatory markers, including CRP and ESR \\ $>$ Urine analysis \\ Chest X-ray \\ Coeliac screen \\ $>$ Stool sample \\ Autoimmune screen \\ Coagulation profile and antiphospholipid antibodies \\ Mouth and genital ulcer swab and culture, occasionally eye swab
}

\section{Other investigations that may be required}

> Oral disease - oral biopsy to exclude orofacial granulomatosis and bullous dermatosis (histology and direct immunofluorescence)

> Genital disease - vulval biopsy to exclude mimics (eg lichen sclerosis)

> Neuro and vascular BD - Doppler studies, CT or MRI brain and spinal cord, MRV/CTV, MRA or CTA to evaluate neurologic and vascular disease; CSF studies, EEG, EMG, NCS, ${ }^{18} \mathrm{~F}$ fluoro2-deoxyglucose PET with CT/MRI localisation (for early inflammation in large vessel vasculitis)

> Musculoskeletal BD - synovial fluid analysis, X-ray, ultrasound or MRI to evaluate joints

> Skin BD - skin biopsy and immunofluorescence

> Cardiac BD - electrocardiogram, echocardiogram

> Chest CT - assess mediastinal diseases, fibrosing mediastinitis, aneurysms, pleural effusions, complications of venous thrombosis and collaterals

> Gastrointestinal BD - stool sample for faecal calprotectin, endoscopy and biopsy

$>$ Ear, nose and throat disease: nasal endoscopy

Ocular BD - OCT, VEP, fluorescein angiogram, Schirmer's test, intraocular fluid culture to exclude infections

> Cystoscopy and kidney, urinary bladder CT for urological disease

$\mathrm{BD}=$ Behçet's disease; $\mathrm{CRP}=\mathrm{C}$-reactive protein; $\mathrm{CSF}=$ cerebrospinal fluid; $\mathrm{CT}$ = computerised tomography; CTA = computerised tomography angiography; $\mathrm{CTV}$ = computerised tomographic venography; $\mathrm{EEG}=$ electroencephalogram; $E M G$ = electromyography; $E S R$ = erythrocyte sedimentation rate; $M R A=$ magnetic resonance angiography; $\mathrm{MRI}=$ magnetic resonance imaging; $\mathrm{MRV}=$ magnetic resonance venography; NCS = nerve conduction study; $\mathrm{OCT}=$ optical coherence tomography: $\mathrm{PET}=$ positron emission tomography: $\mathrm{VEP}=$ visual evoked potentials

(50-100 mg in $10 \mathrm{~mL}$ of water) used 3-4 times daily as mouthwash are particularly effective, suggesting a role of oral bacteria in ulcer pathogenesis.

Benzydamine hydrochloride $0.15 \%$ oral rinse (local analgesic), chlorhexidine mouthwash (anti-infective) and lidocaine spray or gel are useful adjuncts in symptom management. A meta-analysis of interventions for oral ulcers in $\mathrm{BD}$ failed to either confirm or refute the effectiveness of various interventions because of the paucity of good quality studies. ${ }^{23}$
Table 3. Disease modifying drugs (DMDs) used in

Behçet's disease

\section{Oral DMDs}

Azathioprine

Mycophenolate mofetil

Methotrexate

Tacrolimus

Ciclosporin

Sulfasalazine

Dapsone

Thalidomide (exceptional

use)

Colchicine

Prednisolone

\section{Parenteral treatment}

Cyclophosphamide

\section{Anti-TNF $\alpha$ inhibitors}

Infliximab

Adalimumab

Etanercept

Certolizumab

Rituximab

Interferon $\alpha$

Alemtuzumab

\section{Dose of drug}

$2-3 \mathrm{mg} / \mathrm{kg} / \mathrm{day}$

2-3 gram/day

20-25 mg/week

4-8 mg/day (depending on plasma trough levels)

$2-5 \mathrm{mg} / \mathrm{kg} / \mathrm{day}$

$2-3$ g/day

$2-3 \mathrm{mg} / \mathrm{kg} /$ day

$50-300 \mathrm{mg} / \mathrm{day}$

0.5-2 mg/day

Variable dose (depending on indication and stage of treatment)

$15 \mathrm{mg} / \mathrm{kg}$ (vasculitis regimens)

$5 \mathrm{mg} / \mathrm{kg}$ at 0,2 and 6 weeks then once every 8 weeks

$40 \mathrm{mg}$ every 2 weeks

$50 \mathrm{mg} /$ week

$400 \mathrm{mg}$ at 0,2 and 4 weeks then once every 4 weeks

$1 \mathrm{~g}$ at 0 and 2 weeks

Various regimens for Roferon $\mathrm{A}$ and pegylated interferon $\alpha 2 b$

$3 \mathrm{mg}$ (day 1 ), $10 \mathrm{mg}$ (day 3), $30 \mathrm{mg}$ (day 5), $30 \mathrm{mg}$ (day 8 ), $30 \mathrm{mg}$ (day 10 ) and $30 \mathrm{mg}$ (day 12)
Genital aphthous ulcers are severely painful and should be treated with strong analgesics, topical anaesthetics (10\% lidocaine spray or $2-5 \%$ gel), topical corticosteroid ointment (dermovate) or paste, oral corticosteroids and antibiotics in case of secondary infection of the genital ulcer. Intralesional steroids may be offered in intractable ulcers. Skin barrier can be maintained with emollients. General vulval care, avoiding soaps that can cause irritation of vulval mucosa and use of soap substitutes (dermol) are often reported to be useful by patients. Care should be taken to avoid injury to vulval and perineal skin to avoid the pathergy phenomenon, infection and pseudofolliculitis.

\section{Box 3. Newer medications on the horizon (further}

\section{evidence needed)}

$$
\begin{aligned}
> & \text { Apremilast - phosphodiesterase-4 inhibitor }{ }^{18} \\
> & \text { Anakinra - interleukin (IL)-1 receptor antagonist } \\
& \\
> & \text { Canakinumab - human monoclonal immunoglobulin G1 } \\
& \text { inhibitor of IL-1 } \beta^{20} \\
> & \text { Tocilizumab - IL-6 inhibitor }{ }^{21} \\
> & \text { Ustekinumab - IL-12/23 inhibitor }
\end{aligned}
$$


Cutaneous lesions are treated topically with steroid creams and oral antibiotics such as lymecycline, which has anti-inflammatory properties. Erythema nodosum-like lesions usually respond to colchicine $(0.5-2 \mathrm{mg} / \mathrm{d}$, orally) and corticosteroids. Colchicine, which offers significant benefit in arthritis and mucocutaneous disease, exerts its anti-inflammatory activity by inhibiting neutrophil chemotaxis. ${ }^{24}$ Non-responsive mucocutaneous disease requires escalation of treatment to non-biologic diseasemodifying drugs (DMDs). Pyoderma gangrenosum is intensely inflammatory and requires strong immunosuppression with steroids and DMDs.

Azathioprine (2-3 mg/kg/day orally), which inhibits DNA synthesis, is beneficial at doses higher than normally used in other rheumatic diseases. ${ }^{25,26}$ Mycophenolate mofetil (MMF; 2-3 gm/day orally) is an inhibitor of T- and B-lymphocytes. Dapsone (2-3 mg/kg/day), through its anti-inflammatory and bactericidal properties, has a role in cutaneous disease. ${ }^{27}$ Checking G6PD deficiency is important prior to dapsone use, to avoid haemolysis. TNF- $\alpha^{28-34}$ and interferon $\alpha^{35-40}$ are potent biological drugs used in resistant mucocutaneous disease. Thalidomide, ${ }^{41}$ a TNF inhibitor, is now used only in exceptional circumstances (if other DMDs and/or biologics have failed), but needs extremely careful monitoring because of its very narrow therapeutic index. Nerve conduction studies must be performed at baseline and then serially and after each dose change of thalidomide to detect potential neurotoxicity; the drug should be immediately discontinued if symptoms suggesting peripheral neuropathy are reported. Women of childbearing age must have pregnancy excluded prior to its use and agree to use foolproof contraception, with regular pregnancy tests while on the drug.

Musculoskeletal symptoms are managed with various analgesia, including non-steroidal anti-inflammatory drugs (where appropriate), as well as physical therapy. The inflammatory symptoms are suppressed with oral DMDs, escalating to biologics (typically TNF inhibitors). Fibromyalgia is frequently observed in $\mathrm{BD}$. It requires an approach of graded physical activity, pain-modifying medications (eg amitriptyline, pregabalin) and cognitive behavioural therapy. Enthesial inflammation may be amenable to local steroid injections and physical therapy and, if not, DMDs.

Inflammatory eye disease must be managed in partnership with an experienced ophthalmologist. Treatment involves tailoring therapy to the site of disease and impending threat of visual loss. Anterior uveitis is treated with topical steroids, cycloplegics and mydriatics with addition of oral steroids $40 \mathrm{mg}$ average for 4-6 weeks and extending to 3 months if required. Posterior uveitis is suppressed with steroids and DMDs like azathioprine (used in higher doses than other rheumatic diseases, $2.5-3 \mathrm{mg} / \mathrm{kg}$ ) or ciclosporin; ${ }^{42}$ MMF may be used as a second-line drug with beneficial effects noted in cystoid macular oedema. Retinal vasculitis is aggressively treated with steroids (intravenous, oral, intraocular) and DMDs. For resistant and/or aggressive disease, rapid escalation to biologics therapy with infliximab $(5 \mathrm{mg} / \mathrm{kg}$ at 0,2 and 6 weeks then once every 6 weeks thereafter) or interferon alpha, which is a potent drug; DMDs should be bypassed if indicated. Refractory disease is managed with rituximab ${ }^{43}$ or alemtuzumab.

Vascular BD is treated with DMDs and steroids. The arterial aneurysm requires strong immunosuppressants. Intravenous steroids (three pulses of 1,000 mg methylprednisolone) followed by oral steroids and cyclophosphamide are used to rapidly control the disease. Oral DMDs (azathioprine) can be used 6 months after induction with cyclophosphamide. Alternatively, TNF inhibitors can be used in place of cyclophosphamide.

Enlarging arterial aneurysm is an emergency that will necessitate consideration of endovascular repair to avert rupture or pressure effect. ${ }^{44,45}$ Open surgical repair carries a high risk of morbidity and mortality and is complicated by the formation of pseudoaneurysms. ${ }^{46}$ All surgical and endovascular interventions should be supported with heavy immunosuppression. Expert opinion is divided on the treatment of deep venous thrombosis and cerebral venous sinus thrombosis: some experts recommend anticoagulation, while others believe that effective immunosuppression is key. ${ }^{17}$ A 2015 observational study did not show any favourable effect from the addition of anticoagulation to immunosuppresion on the relapse rates of vascular events in BD. ${ }^{47}$ We strongly advocate intense immunosuppression, which is required to reduce the stimulus for thrombosis and do not recommend anticoagulation, unless detailed vascular imaging has excluded the presence of arterial aneurysms. However, anticoagulation may be indicated in the presence of another pro-thrombotic condition (eg Protein C and S resistance, Factor V Leiden) but, again, coexisting arterial aneurysms must be excluded. Stenotic arterial lesions require stenting to help re-establish perfusion. Pulmonary aneurysms can be coiled and bleeding vessels embolised. Bronchial artery embolisation is frequently required as it is the commonest cause of bleeding and haemoptysis.

Neuro BD (parenchymal and non-parenchymal) is managed with strong immunosuppression, as with other systemic involvement in BD. ${ }^{11}$ Intravenous cyclophosphamide along with high-dose intravenous methylprednisolone (1 g daily for 3 days) followed by oral corticosteroids can be used in neurovascular diseases prior to TNF inhibitors, but ciclosporin is generally avoided. The sequelae of neurological damage require physical therapy and neuro-cognitive assessment and support. Management of migraine is similar to subjects without BD. This requires lifestyle changes like good hydration, sleep hygiene, dietary modifications and medications (acute medications like triptans and preventatives such as propranolol, nortriptyline or topiramate).

Gastrointestinal BD presents like IBD and is treated similarly with immunosuppresssion with local and oral steroids and both non-biological (salazopyrin or azathioprine) and biological DMDs. The complications of perforation and fistulation require surgical intervention supported by strong immunosuppression.

A head-to-head trial (BIO BEHCET'S) of infliximab with interferon $\alpha$ is underway, ${ }^{48}$ which will demonstrate the positional hierarchy of the above treatment in $\mathrm{BD}$ and also provide important genetic and biomarker clues to BD. ${ }^{48}$

In pregnancy, cyclophosphamide, methotrexate, MMF and thalidomide are contraindicated. Although we do not recommend colchicine, studies have not shown adverse outcomes. ${ }^{49}$ The safety of biological agents in $\mathrm{BD}$ pregnancy is extrapolated from its use in other rheumatic diseases and balanced against risk-benefit considerations. Use of biologic agents other than TNF inhibitors in BD is not well known. Non-steroidal anti-inflammatory drugs should be avoided in the third trimester of pregnancy because of the risk of premature closure of the patent ductus arteriosus. 
Hematopoietic stem cell transplantation has been used with success in severe BD that had not responded to non-biological and biological DMDs ${ }^{50,51}$ and the European Bone Marrow Transplant registry (www.ebmt.org) has data on its use and benefits. $^{52}$

Measurement of the disease activity with disease-specific measures (Behçet's Disease Current Activity Form (BDCAF) ${ }^{53}$ and the Leeds BD-quality of life (BD-QoL) ${ }^{54}$ ) and organ-specific measures and impact scores (Oral Ulcer Activity Index, ${ }^{55}$ The Expanded Disability Status Scale, the modified Rankin scale ${ }^{56}$ and neuro-Behçet's disability score) is important. Quality of life measures like the health assessment questionnaire disability index, short form health survey 36 and the EQ-5D-5L are important to assess the impact of disease and the effects of intervention.

\section{Prognosis}

The impact of BD is significant, with effects on somatic and psychosocial wellbeing. The organ-specific morbidity is high and arterial disease influences mortality. ${ }^{57}$ While there is no direct effect on fertility, the influence of genital ulcerations and psychological stress is immense. The patient's ability to work is also adversely influenced in BD.

\section{Follow-up}

Patients with BD should be offered a multidisciplinary assessment and management plan. Furthermore, follow-up under a physician with guidance from a specialist centre will be important for overview of disease and management. Organ-specific flares will require relevant specialist input. Patients should be encouraged to play a large role in self-care, which is important in chronic conditions. Relevant input from allied specialties, like physical and occupational therapists, psychologists and social support workers, is vital.

\section{Conclusions}

$\mathrm{BD}$ is a chronic, relapsing vasculitis with potential to affect all body systems with profound effects, causing considerable morbidity and mortality. There is a delay in diagnosis because of variable symptoms and a lack of valid biomarkers. A number of biomarkers have been described but none have proven to be reliable or valid for application in clinical practice for diagnosis, assessment of disease activity and monitoring. The research agenda for $\mathrm{BD}$ is huge, as so little is known. This ranges from basic science explorations into the potential pathophysiological mechanisms underlying the disease (if we don't know the cause, it's not surprising that treatment is not that good), through a better phenotyping of the clinical situation to more effective treatment. On the treatment side, there are two pressing issues:

1 knowing how and when treatment, especially with biologics, can be de-escalated or stopped

2 the development of biomarkers allowing a personalised medicines approach to therapy.

Finally, the other major research agenda is the development of an effective disease activity score and enhanced patientreported outcome tool. There is a large unmet need for novel therapy in $\mathrm{BD}$, which will hopefully become available as the aetiopathogenetic mechanisms are unlocked.

\section{Conflicts of interest}

The authors have no conflicts of interest to declare.

\section{References}

1 Behcet $\mathrm{H}$. Uber rezidiverendeaphthose durch ein virus verursachte Geschwure am Mund, am Auge, und an den Genitalien. Dermatol Wochenschr 1937;105:1152-7.

2 Yurdakul S, Gunaydin I, Tuzun Y et al. The prevalence of Behcet's syndrome in a rural area in northern Turkey. J Rheumatol 1988;15:820-2.

3 Chamberlain MA. Behçet syndrome in 32 patients in Yorkshire. Ann Rheum Dis 1977;36:491-9.

4 Yazici H, Chamberlain A, Schreuder I et al. HLA antigens in Behçet's disease: a reappraisal by a comparative study of Turkish and British patients. Ann Rheum Dis 1980;39:344-8.

5 Remmers EF, Cosan F, Kirino Y et al. Genome-wide association study identifies variants in the MHC class I, IL10, and IL23R-IL12RB2 regions associated with Behçet's disease. Nat Genet 2010;42:698-702.

6 Mizuki N, Meguro A, Ota M et al. Genome-wide association studies identify IL23R-IL12RB2 and IL10 as Behçet's disease susceptibility loci. Nat Genet 2010;42:703-6.

7 Kirino Y, Bertsias G, Ishigatsubo Y et al. Genome-wide association analysis identifies new susceptibility loci for Behçet's disease and epistasis between HLA-B ${ }^{\star} 51$ and ERAP1. Nat Genet 2013;45:202-7.

8 International Team for the Revision of the International Criteria for Behcet's Disease. Revision of the International Criteria for Behcet's Disease (ICBD). Clin Exp Rheumatol 2006;24(Suppl 42):S14-5.

9 International Team for the Revision of the International Criteria for Behçet's Disease (ITR-ICBD). The International Criteria for Behçet's Disease (ICBD): a collaborative study of 27 countries on the sensitivity and specificity of the new criteria. J Eur Acad Dermatol Venereol 2014;28:338-47.

10 International Study Group for Behçet's Disease. Criteria for diagnosis of Behçet's disease. International Study Group for Behçet's Disease. Lancet 1990;335:1078-80.

11 Kalra S, Silman A, Akman-Demir G et al. Diagnosis and management of Neuro-Behçet's disease: international consensus recommendations. J Neurol 2014;261:1662-76.

12 Tugal-Tutkun I, Onal S, Altan-Yaycioglu R, Huseyin Altunbas H, Urgancioglu M. Uveitis in Behçet disease: an analysis of 880 patients. Am J Ophthalmol 2004;138:373.

13 Jadaon J, Shushan A, Ezra Y et al. Behçet's disease and pregnancy. Acta Obstet Gynecol Scand 2005;84:939-44.

14 Iskender C, Yasar O, Kaymak O et al. Behcet's disease and pregnancy: a retrospective analysis of course of disease and pregnancy outcome. J Obstet Gynaecol Res 2014;40:1598-602.

15 Gilio $M$ et al. Behcet's disease and pregnancy: what is the relationship? Ann Rheum Dis 2015;74(Suppl 2):516.

16 Hughes JP, Stovin PG. Segmental pulmonary artery aneurysms with peripheral venous thrombosis. Br J Dis Chest 1959;53:19-27.

17 Hatemi G, Silman A, Bang D et al. EULAR recommendations for the management of Behçet disease. Ann Rheum Dis 2008;67:1656-62.

18 Hatemi G, Melikoglu M, Tunc R et al. Apremilast for Behcet's syndrome - A phase 2, placebo-controlled study. N Engl J Med 2015;372:1510-8.

19 Botsios C, Sfriso P, Furlan A, Punzi L, Dinarello CA. "Resistant Behçet disease responsive to anakinra." Ann Intern Med 2008;149:284-6.

20 Vitale A, Rigante D, Caso F et al. Inhibition of Interleukin-1 by canakinumab as a successful mono-drug strategy for the treatment of refractory Behçet's disease: a case series. Dermatology 2014;228:211-4.

21 Hirano T et al. A case of Behçet's disease treated with a humanized anti-interleukin-6 receptor antibody, tocilizumab. Modern Rheumatology 2012;298-302. 
22 Baerveldt EM, Kappen JH, Thio HB et al. Successful long-term triple disease control by ustekinumab in a patient with Behçet's disease, psoriasis and hidradenitis suppurativa. Ann Rheum Dis 2013;72:626-7.

23 Taylor J, Glenny A, Walsh T et al. Interventions for the management of oral ulcers in Behçet's disease. Cochrane Database Syst Rev 2014;(9):CD011018.

24 Yurdakul S, Mat C, Tüzün Y et al. A double-blind trial of colchicine in Behçet's syndrome. Arthritis Rheum 2001;44:2686-92.

25 Yazici H, Pazarli H, Barnes CG et al. A controlled trial of azathioprine in Behçet's syndrome. N Engl J Med 1990;322:281-5.

26 Hamuryudan V, Ozyazgan Y, Hizli N et al. Azathioprine in Behçet's syndrome: effects on long-term prognosis. Arthritis Rheum 1997;40:769-74.

27 Sharquie KE, Najim RA, Abu-Raghif AR. Dapsone in Behçet's disease: a double-blind, placebo-controlled, cross-over study. J Dermatol 2002;29:267-79.

28 Melikoglu M, Fresko I, Mat C et al. Short-term trial of Etanercept in Behcet's disease: a double blind, placebo controlled study. J Rheumatol 2005;32:98-105.

29 Estrach C, Mpofu S, Moots RJ. Behçet's syndrome: response to infliximab after failure of etanercept. Rheumatology 2002;41:1213-4.

30 Arida A, Fragiadaki K, Giavri E, Sfikakis PP. Anti-TNF agents for Behcet's disease: analysis of published data on 369 patients. Semin Arthritis Rheum 2011;41:61-70.

31 Pipitone N, Olivieri I, Padula A et al. Infliximab for the treatment of Neuro-Behçet's disease: a case series and review of the literature. Arthritis Rheum 2008;59:285-290.

32 Al-Araji A, Siva A, Saip S et al. Treatment of Neuro Behçet's disease with infliximab. An international multi-centre case-series of 18 patients. Clin Exp Rheumatol 2010;28:S119.

33 Fasano A, D’Agostino M, Caldarola G et al. Infliximab monotherapy in neuro-Behçet's disease: four year follow-up in a long-standing case resistant to conventional therapies. J Neuroimmunol 2011;239:105-7.

34 Olivieri I, Leccese P, D’Angelo S et al. Efficacy of adalimumab in patients with Behçet's disease unsuccessfully treated with infliximab. Clin Exp Rheumatol 2011;29:S54-7.

35 Alpsoy E, Durusoy C, Yilmaz E et al. Interferon $\alpha-2 \mathrm{a}$ in the treatment of Behçet disease: a randomized placebo-controlled and double-blind study. Arch Dermatol 2002;138:467-71.

36 Kotter I, Vonthein R, Zierhut M et al. Differential efficacy of human recombinant interferon- $\alpha 2 \mathrm{a}$ on ocular and extraocular manifestations of Behçet disease: results of an open 4-center trial. Semin Arthritis Rheum 2004;33:311-9.

37 Wechsler B, Bodaghi B, Huong DL et al. Efficacy of interferon $\alpha-2 \mathrm{a}$ in severe and refractory uveitis associated with Behçet's disease. Ocul Immunol Inflamm 2000;8:293-301.

38 Bodaghi B, Gendron G, Wechsler B et al. Efficacy of interferon $\alpha$ in the treatment of refractory and sight threatening uveitis: a retrospective monocentric study of 45 patients. Br J Ophthalmol 2007;91:335-9.

39 Kotter I, Gunaydin I, Zierhut M, Stubiger N. The use of interferon $\alpha$ in Behçet disease: review of the literature. Semin Arthritis Rheum 2004;33:320-35.

40 Lightman S, Taylor SJ, Bunce C et al. Pegylated interferon- $\alpha-2 b$ reduces corticosteroid requirement in patients with Behçet's disease with upregulation of circulating regulatory $\mathrm{T}$ cells and reduction of Th17. Ann Rheum Dis 2015;74:1138-44.

41 Hamuryudan V, Mat C, Saip S et al. Thalidomide in the treatment of the mucocutaneous lesions of the Behcet syndrome. A randomized, double-blind, placebo-controlled trial. Ann Intern Med 1998;128:443-50.
42 BenEzra D, Cohen E, Chajek T et al. Evaluation of conventional therapy versus cyclosporine A in Behçet's syndrome. Transplant Proc 1988;20(Suppl 4):136-43.

43 Davatchi F, Shams H, Rezaipoor M et al. Rituximab in intractable ocular lesions of Behcet's disease; randomized single-blind control study (pilot study). Int J Rheum Dis 2010;13:246.

44 Nitecki SS, Ofer A, Karram T et al. Abdominal aortic aneurysm in Behçet's disease: new treatment options for an old and challenging problem. Isr Med Assoc J 2004;6:152-5.

45 Park JH, Chung JW, Joh JH et al. Aortic and arterial aneurysms in Behçet disease: management with stent-grafts - initial experience. Radiology 2001;220:745-50.

46 Hosaka A, Miyata T, Shigematsu H et al. Long-term outcome after surgical treatment of arterial lesions in Behçet disease. J Vasc Surg 2005;42:116-21.

47 Alibaz-Oner F, Karadeniz A, Ylmaz S et al. Behçet disease with vascular involvement: effects of different therapeutic regimens on the incidence of new relapses. Medicine 2015;94:e494.

48 Optimal utilisation of biologic drugs in Behçet's Disease: a randomised controlled trial of infliximab (IFX) verses alpha interferon (aIFN), with genotyping and metabolomic profiling, towards a stratified medicines approach to treatment. www.hra. nhs.uk/news/research-summaries/bio-behcets/ [Accessed 20 December 2016].

49 Diav-Citrin O, Shechtman S, Schwartz V et al. Pregnancy outcome after in utero exposure to colchicine. Am J Obstet Gynecol 2010;203:144.e1-6.

50 Chauhan S, Olujohungbe A, Moots RJ. Treatment of Behçet's syndrome and plasmacytoma in a patient with peripheral blood stem cell transplantation. Leuk Lymphoma 2008;49:2377-9.

51 Soysal T, Salihoglu A, Esatoglu SN et al. Bone marrow transplantation for Behçet's disease: a case report and systematic review of the literature. Rheumatology 2014;53:1136-41.

52 Farge D, Labopin M, Tyndall A et al. Autologous hematopoietic stem cell transplantation for autoimmune diseases: an observational study on 12 years' experience from the European Group for Blood and Marrow Transplantation Working Party on Autoimmune Diseases. Haematologica 2010;95:284-92.

53 Bhakta B, Brennan P, James T et al. Behcet's disease: evaluation of a new instrument to measure clinical activity. Rheumatology 1999;38:728-33.

54 Gilworth G, Chamberlain MA, Bhakta B et al. Development of the BD-QoL: a quality of life measure specific to Behcet's disease. J Rheumatol 2004;31:931-7.

55 Mumcu G, Sur $\mathrm{H}$, Inanc $\mathrm{N}$ et al. A composite index for determining the impact of oral ulcer activity in Behcet's disease and recurrent aphthous stomatitis. J Oral Pathol Med 2009;38:78591.

56 Bonita R, Beaglehole R. Modification of Rankin scale: recovery of motor function after stroke. Stroke 1988;19:1497-500.

57 Saadoun D, Wechsler B, Desseaux K et al. Mortality in Behcet's disease. Arthritis Rheum 2010;62:2806-12.

Address for correspondence: Dr J R Nair, Aintree University Hospital, Lower lane, Liverpool, Merseyside L9 7AL, UK. Email: jagdish.nair@aintree.nhs.uk 